\title{
L'intégration économique de la Méditerranée par les réseaux maritimes et portuaires ${ }^{1}$
}

\author{
Nora MAREÏ, CNRS \\ César DUCRUET, CNRS \& UMR 8504 Géographie-cités
}

Ceci est la version pré-finale de l'article publié dans la revue Maghreb-Mashrek, No. 220, pp 17-33

Résumé :

Suite au percement du canal de Suez à la fin du XIX ${ }^{\mathrm{e}}$ siècle, la Méditerranée devient un carrefour maritime majeur entre Europe, Asie et Afrique. La région portuaire connait dès lors un développement rapide et extraverti qui s'est accéléré ces dernières décennies, sous référence d'une nouvelle étape de la mondialisation. La participation des pays du Sud à cette économie mondialisée est le signe d'évolutions profondes que nous proposons d'aborder, d'une part à travers l'étude des réseaux maritimes et portuaires, et d'autre part à travers une réflexion plus conceptuelle sur l'intégration régionale et l'enracinement territorial des processus mondialisés.

Mots-clefs : Méditerranée, Ports, Circulations, Réseaux, Régionalisation, Intégration

Lieu d'éclatement logistique idéal entre Europe, Afrique et Asie, la Méditerranée ne cesse de renforcer ses positions sur la scène maritime internationale. Les ports se modernisent et s'internationalisent au rythme des extensions portuaires et de l'installation d'acteurs économiques internationaux de la logistique et de l'industrie ( $c f$. figure 1). Les circulations qui en découlent et les stratégies territoriales mises en œuvre pour capter les flux de marchandises et d'investissements participent à la fabrique d'une régionalisation nouvelle, fortement empreinte de mondialisation et dont les effets locaux méritent d'être discutés. Outre la modernisation des infrastructures portuaires, la mise en réseau croissante des ports méditerranéens est le signe d'une recomposition des logiques régionales. Si ces processus sont généralement orchestrés par des prises d'intérêts extérieures marquant un renforcement de l'extraversion des économies méditerranéennes, nous posons l'hypothèse qu'ils participent néanmoins à une régionalisation économique d'une part, et à une intégration plus globale du bassin d'autre part. Ces considérations nous incitent à proposer un cadre d'analyse voisin de celui appliqué par François Gipouloux (2009) à la Méditerranée asiatique et à nous demander en quoi un espace maritime régional fait système tant au niveau des acteurs que des flux et des territoires connectés.

\footnotetext{
${ }^{1}$ Cet article a été réalisé dans le cadre du projet World Seastems financé par le Conseil Européen de la Recherche (European Research Council), FP7-IDEAS-ERC Starting Grant No. 313847
} 
Figure 1 - Trafics conteneurisés des ports méditerranéens (2012)

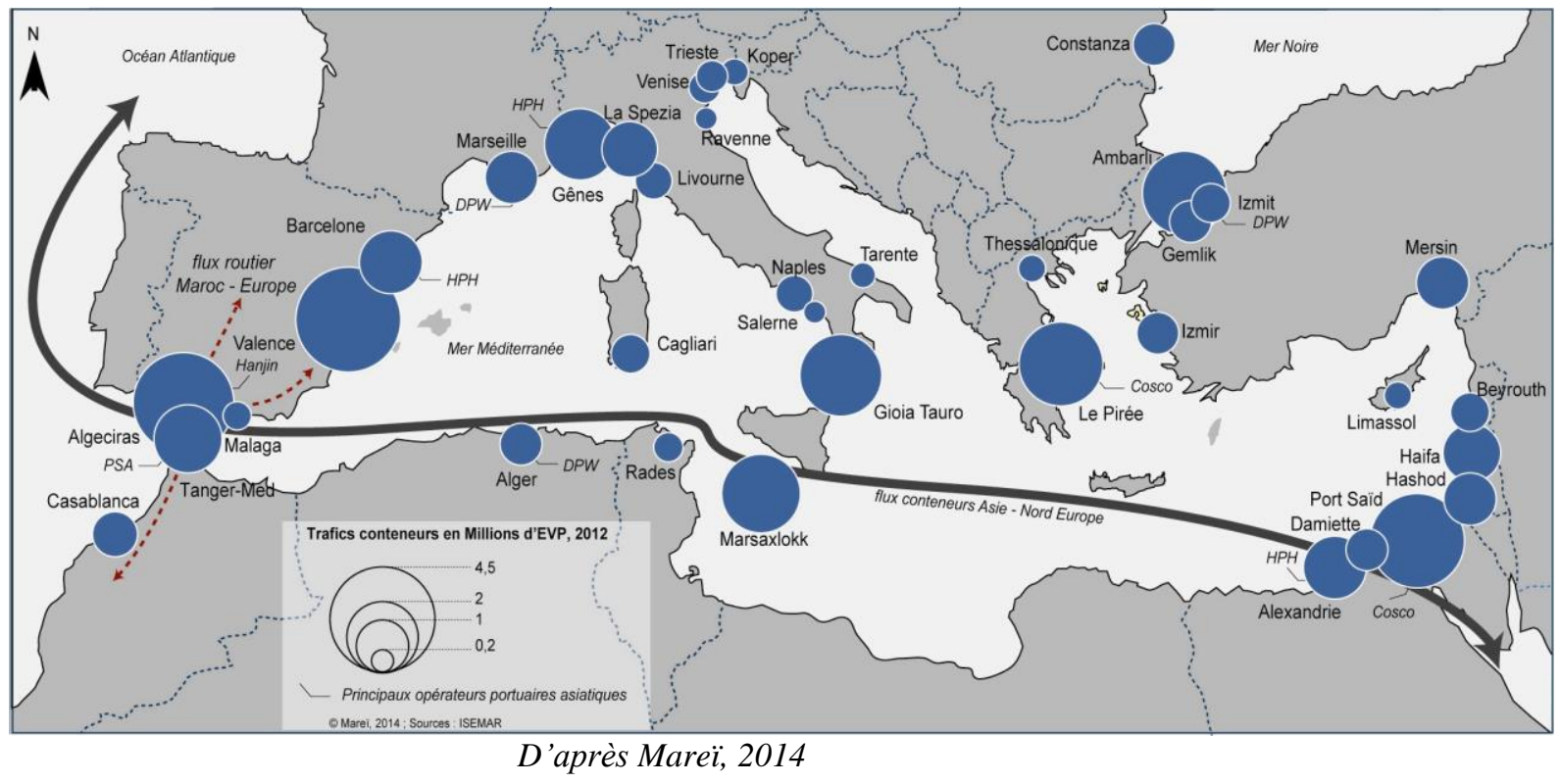

Il convient tout d'abord de préciser de quelles formes d'intégration et de régionalisation nous souhaitons discuter. En effet, tout comme la région, forme spatiale à laquelle ces processus se rapportent, intégration et régionalisation demeurent des concepts polysémiques évoluant vers une acception plurielle et multi-scalaire dans la littérature récente en géographie. La région dans la mondialisation de l'économie transcende les Etats-nations et devient selon Kenichi Ohmae (1996) une «unité opérationnelle naturelle de l'économie planétaire contemporaine ». Mobilités et circulations sont les grands paradigmes de cette mondialisation et semblent trouver leur pleine expression dans une échelle régionale située quelque part entre le local et le global. Le «new regionalism ( Hettne et Söderbaum, 1998) y voit un processus extraverti dont les limites sont floues de facto, nécessitant par là une approche régionale globale et pluridisciplinaire. C'est également le cas de Martin Jones et Anssi Paasi (2013) qui insistent sur le « rescaling » permis par l'approche régionale, ou encore d'Ash Amin (2004) qui présente la région comme l'expression d'une connectivité spatialisée. Ces approches s'opposent aux études classiques de la région de Paul Vidal de la Blache (1910) à Paul Claval (1968), dans lesquelles la région est mise en catégories (urbaine, nodale, en déclin...) et elle est surtout d'échelle sub-nationale. Cette question de l'échelle demeure essentielle au sein du débat régional et les échelles supra, méso ou encore macro deviennent des passages nécessaires pour appréhender les territoires dans la mondialisation. Un certain nombre de travaux revendiquent ainsi une échelle macro-régionale, composée de pays proches les uns des autres (Richard, 2010), et qui analysent de «grandes régions» (Girault, 2009), des «régions Nord-Sud» (Beckouche, 2008), ou encore des continents (Carroué, 2007; Grataloup, 2009).

En lien avec le transport maritime, la question régionale reste peu explorée même si elle se retrouve de façon implicite dans les travaux pionniers d'André Vigarié (1964) sur la rangée Nord-Européenne. Une rangée maritime est définie comme un ensemble de ports voisins, devenant interdépendants du fait de leur insertion dans des services maritimes communs de grands armateurs (services pendulaires, boucles), mais aussi par le recouvrement au moins partiel de leurs arrière-pays terrestres. A ces critères, Arnaud Lemarchand et Olivier Joly (2009) ajoutent celui, essentiel, des échanges de données informatisées (EDI) qui complètent 
l'intégration des flux matériels au sein d'un groupe de ports. Lemarchand et Joly (2009) vont même jusqu'à comparer les dynamiques de trafic (conteneurisé) de diverses rangées et concluent à des niveaux très variables de maturité de ces systèmes régionaux. La plupart du temps, les travaux de géographie maritime et portuaire sur des espaces régionaux à dimensions variables tiennent pour acquis les contours géographiques de l'espace étudié, et n'abordent que très rarement la configuration concrète des flux parcourant ces espaces. Le flou terminologique caractérisant les concepts de région portuaire, région maritime, façade, rangée, etc. qui perdure à l'heure actuelle est emblématique de cet état de fait.

La différence entre région portuaire, groupe de ports situés en proximité au sein d'une aire géographique donnée (Ducruet, 2009), et région maritime - que nous définissons comme un ensemble au sein duquel sont analysés les interactions réciproques et les liens entre la mosaïque de territoires et d'échelles - est proche de celle qui existe entre régionalisation et intégration régionale. La première peut ainsi être définie comme l'émergence de pratiques régionales alors que la seconde caractérise un processus plus global, qui se construit dans le temps et qui incorpore dans un «tout régional » un ensemble de processus distincts. Cette vision globale se retrouve chez Björn Hettne et Fredrik Söderbaum (2000) qui définissent l'intégration régionale comme le fruit d'un processus de régionalisation dynamique, «in the making », qui se construit à la fois par le haut et par le bas. Ils proposent le terme de Regionness pour décrire la formation de la région comme un processus évolutif, voire élastique, cette notion étant retrouvée chez Jones et Paasi lorsqu'ils précisent que «regions are seen to stretch in space ».

Une initiative provenant du domaine des Area Studies américaines attire notre attention car elle propose, dans la perspective de renouveler ce champ disciplinaire, de focaliser les approches régionales sur des bassins maritimes (Bentley, 1999; Lewis et Wigen, 1999). Les auteurs considèrent les bassins maritimes comme des zones de contact entre différentes sociétés littorales qu'il s'agit d'analyser de façon pluri-thématiques (politique, historique, culturel, etc.). L'étude de la Ford Foundation (1997) citée par ces auteurs et intitulée Area Studies: Regional Worlds différencie ainsi une "trait geography» d'une «process geography ${ }^{2}$ » rejoignant ainsi le débat sur l'analyse relationnelle des territoires comme nouveau paradigme de la géographie régionale.

C'est dans cette perspective d'une analyse régionale globale et renouvelée que nous souhaitons placer notre réflexion sur un bassin méditerranéen dont l'évolution globale mérite une attention particulière dans le cadre d'une réflexion sur les intégrations en Méditerranée. Nous présentons d'abord le cadre d'une région portuaire transformée par les restructurations globales des transports maritimes, dues en particulier à la généralisation de la conteneurisation. Cette dernière modifie durablement les hiérarchies portuaires du bassin et permet à la rive de Sud de s'ancrer à la mondialisation des échanges. La question de la mer, espace de liens entre les rivages voisins ou simple lieu de transit vers un outre-mer, est ensuite traitée par une analyse de l'évolution des réseaux intra-méditerranéens (1890-2011). Enfin, la tension mondial - local est abordée par une réflexion sur les ancrages territoriaux des processus mondiaux analysés.

\footnotetext{
${ }^{2}$ Process geography fait référence à une géographie relationnelle qui regarde les processus, les acteurs, et les liens entre les différentes composantes des territoires et s'oppose à une Trait geography par essence plus descriptive ou monographique
} 


\section{Une région portuaire au carrefour des échanges mondialisés}

\section{Une mer de nouveau convoitée}

Avec le percement du canal de Suez à la fin du XIX ${ }^{\mathrm{e}}$ siècle, la Méditerranée devient un goulet majeur des routes maritimes entre Europe, Asie et Afrique. Aux grandes routes impériales vers les Indes s'ajoutent après la Seconde Guerre mondiale, une partie de la capture des routes du pétrole du golfe Persique pour les besoins européens, et aujourd'hui celle des flux de produits manufacturés échangés avec l'Extrême-Orient. Il n'est donc pas nouveau de parler de mondialisation en ce qui concerne la Méditerranée, cette dernière étant largement accompagnée d'une maritimisation des économies, rendue visible par la diffusion progressive de l'activité maritime et portuaire ( $c f$. figure 2).

Figure 2 - Evolution de la région portuaire 1890-1961

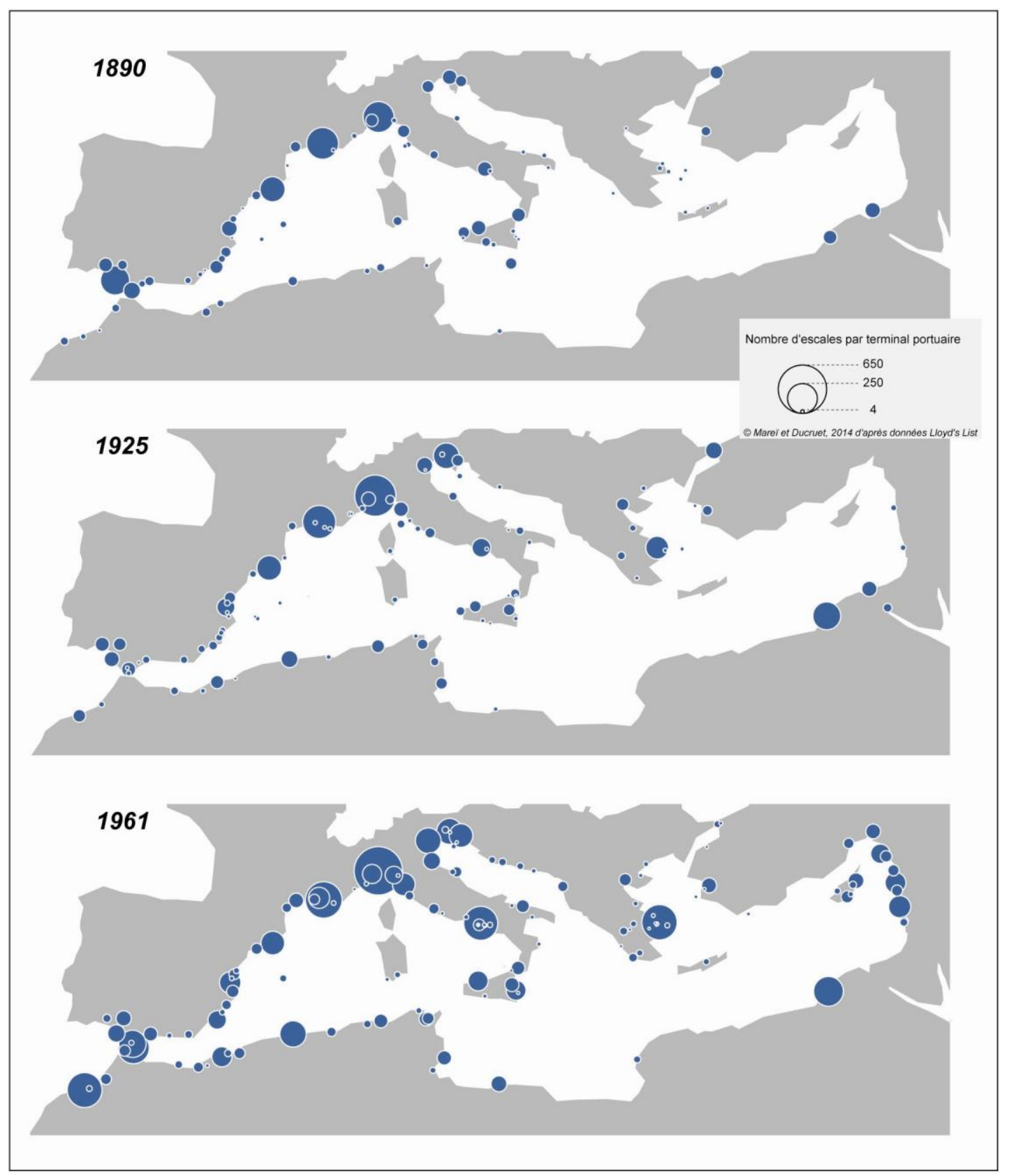

Elaborée par les auteurs à partir de Lloyd's List 
Si les acteurs du jeu géopolitique ont changé à chacune de ces grandes périodes, les lieux stratégiques demeurent. L'Espagne, le Maroc, Gibraltar, Malte, Chypre, l'Egypte, hier têtes de pont et relais des prétentions européennes, sont aujourd'hui des lieux à haute valeur commerciale où se trouvent les principaux $h u b s^{3}$. Les acteurs de cet échiquier sont désormais les grands armateurs et opérateurs maritimes pour qui cette mer reliant l'Europe, l'Afrique et l'Asie est un passage nécessaire par lequel défile une grande partie de la flotte internationale (Vigarié, 1990). Aujourd'hui, 90000 navires fréquentent les eaux du détroit de Gibraltar chaque année, auxquels s'ajoutent les 17500 navires du canal de Suez, et les 40000 qui passent par le Bosphore. Si ces trafics ont crû tout au long du $\mathrm{XX}^{\mathrm{e}}$ siècle, le développement portuaire régional est plus récent. L'implantation d'industrie lourde consolide quelques places portuaires dès les années 1960 (Gênes, Tarente, Marseille-Fos ou encore Algeciras). Cependant, ce ne sont pas les traces de cette industrialisation qui retiennent notre attention, mais plutôt les décisions plus spectaculaires dictées par l'intérêt des acteurs du domaine logistique et marchand pour cette mer à la croisée des routes maritimes mondiales.

L'arrivée de l'armateur Sealand en 1975 à Algeciras marque en effet le point de départ de l'intervention des acteurs mondialisés des transports et de la manutention portuaire en Méditerranée. D'autres lui emboîteront le pas et participeront à la conteneurisation d'abord puis à la mondialisation des réseaux maritimes méditerranéens ensuite : Maersk en 1986 à Algeciras, en 1995 à Gioia Tauro, en 2000 à Port-Saïd, en 2007 à Tanger-Med ; Hutchison Port Holding à Barcelone depuis 2004; Cosco au Pirée depuis 2008, Hanjin Shipping en 2010 à Algeciras, etc. L'arrivée du conteneur en Méditerranée engendre un nouveau contexte pour le frêt maritime qui modifie sur la longue durée les organisations spatiales, économiques et politiques du bassin (Vigarié, 1990, Rodrigue et al., 1997). De ce point de vue, le canal de Suez a joué un rôle décisif, non seulement parce qu'il est bien placé entre l'Europe et l'Asie, mais aussi parce que ses dimensions lui permettent d'accueillir aisément les plus gros porteconteneurs de la planète : son gabarit de 150000 tpl environ en 1980 a été porté, par bonds successifs, à $240000 \mathrm{tpl}$ en 2009. Ainsi, le trafic conteneur de Suez peut être évalué à 398 millions de tonnes en 2011, soit 60\% du trafic total de Suez (contre 26,5\% de trafic conteneurisé à Panama), alors que ce même trafic ne dépassait guère 42 millions de tonnes à Suez en 1985 pour 16,5\% du trafic total du canal (Mareï, 2012).

\section{Les hubs méditerranéens}

Les armements placent leurs hubs sur les routes qui sont les mieux alimentées. Sur la grande route Est-Ouest qui relie les différents pôles de la Triade, on peut estimer qu'en 2007 circulent près de la moitié des boîtes convoyées : 55 millions d'EVP, dont 27,7 millions dans les deux sens des flux entre l'Europe et l'Asie (CNUCED, 2008). C'est donc sur cette route Europe-Asie, et singulièrement en Méditerranée que se développent préférentiellement les hubs (Frémont, 2007). Des terminaux, dont l'activité est artificiellement dopée par la logique des transbordements, ont ainsi vu le jour: Algeciras, Gioia Tauro, Malte (Marsaxlokk), Valence, Le Pirée, Damiette, Port Saïd en font partie, avec des progressions proprement stupéfiantes. Par exemple, Gioia Tauro, ouvert en 1995, enregistre, dix ans plus tard, plus de 3,1 millions d'EVP et talonne Algeciras, un des hubs les plus anciens du bassin. Tanger-Med, inauguré en 2007, enregistre dès 20102 millions d'EVP. Le binôme portuaire du détroit de Gibraltar, Tanger-Med - Algeciras, organisé sur un mode de concurrence / complémentarité

\footnotetext{
${ }^{3}$ Un $h u b$ est une plate-forme d'accueil et d'éclatement de la marchandise vers d'autres destinations.
} 
fait ainsi figure de carrefour méditerranéen à partir duquel interchange (ou interlining : transfert de conteneurs entre gros porteurs à la croisée des routes principales) et feedering (transfert de conteneur entre gros et petits porteurs, grands ports et ports moyens ou secondaires) se déploient vers tous les continents de la planète (Mareï, 2012). Par analogie avec les travaux de Fleming et Hayuth (1994) sur les transports aériens, c'est la position d' « intermédiarité » du détroit et de la Méditerranée qui favorise l'insertion des opérateurs de transport dans les réseaux de la mondialisation, dans «une logique d'espace couloir » voué principalement au transbordement (Ducruet, 2008). Le carrefour de Gibraltar apparaît comme un observatoire de ces bouleversements spatiaux et fonctionnels qui impliquent, en autres, une modification de la gouvernance portuaire en particulier au Sud de la Méditerranée (Ducruet et al., 2011 ; Mohamed-Chérif et Ducruet, 2011): accélération de la vague de privatisation (Tanger-Med sur le modèle de Port-Saïd, est le premier port entièrement privé au Maroc) et implantation croissante des opérateurs asiatiques (arrivée d'Hanjin Shipping à Algeciras, Port of Singapour Authority (PSA) à Tanger-Med II).

L'autre nœud majeur du bassin est situé au débouché septentrional du canal de Suez. Les ports égyptiens de Damiette et Port Saïd sont devenus les hubs incontestés de la Méditerranée orientale. Passages obligés sur la route de Suez, ces ports ont vu leurs trafics augmenter ces dernières années. Autour de Damiette et Port Saïd un réseau dense de lignes feeders alimente les ports israéliens, libanais, syriens et turcs ( $c f$. figure 3). Damiette (0,75 M. d'EVP en 2012 après avoir atteint 1,2 $\mathrm{M}$ en 2011), utilisé comme port d'escale de la CMA-CGM, offre 8 postes à quai et un tirant d'eau de $14 \mathrm{~m}$. Il est également desservi par une pléiade d'armateurs internationaux comme Hapag-Lloyd, Hyundai Merchant Marine ou Maersk depuis le rachat de $\mathrm{P} \& \mathrm{O}$ Nedlloyd. Le port est aujourd'hui dans une phase de modernisation et d'extension, en s'équipant de nouveaux portiques, en développant les trafics rouliers (celui des remorques de camions) et en renforçant sa fonction de plate-forme logistique afin de rester compétitif face au nouveau Suez Canal Container Terminal (SCCT) de Port-Saïd (3,6 M.d'EVP au total en 2012 après un record de 4,3 $\mathrm{M}$ en 2011).

Figure 3 - Un binôme égyptien pour les trafics en transbordement

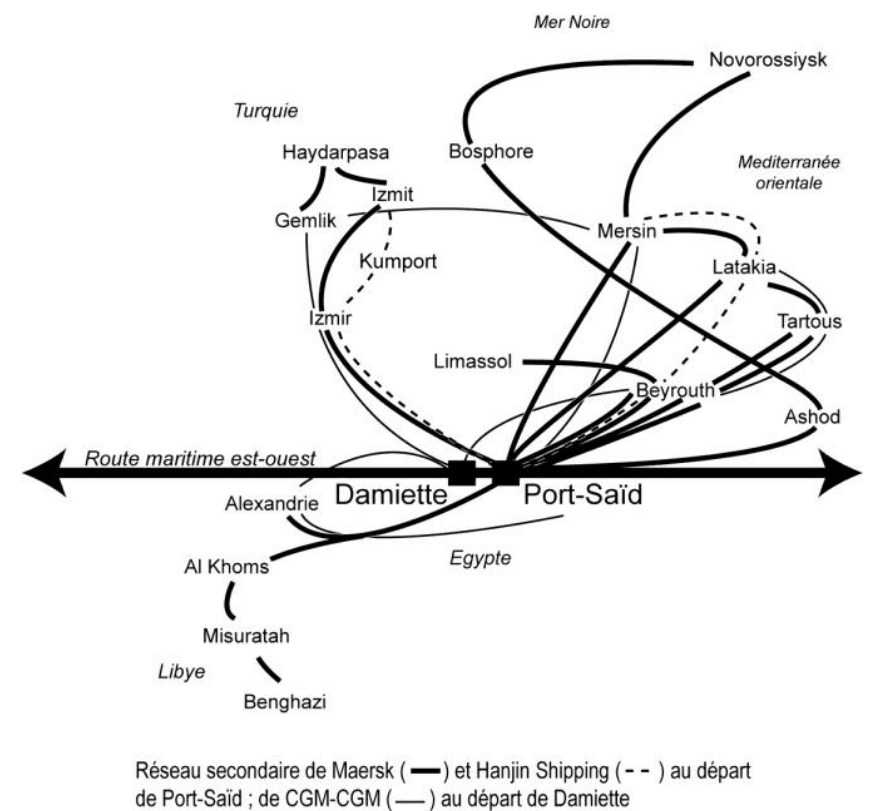

Elaboration N. Mareï 2012 d'après les services feeders maerskline.com, hanjin.com, cma-cgm.fr 
La société du SCCT, qui dispose d'une concession de 30 ans, a été créée en 2000. Elle est actuellement aux mains d'AP Moller Terminals (Danemark) et Cosco (Chine) à hauteur respectivement de $55 \%$ et $20 \%$, le solde étant détenu par les Egyptiens. La première partie de ce terminal, achevée en 2004, comprend un quai de $1200 \mathrm{~m}$ et de 60 ha pour une capacité de 2,7 M d'EVP et accueille douze portiques superpostpanamax ${ }^{4}$. La seconde partie du terminal, dont les travaux se sont achevés en 2012, permet de doubler les capacités portuaires. Ce nouveau port en eau profonde se hisse désormais au premier rang des ports à conteneurs de la Méditerranée et de l'Afrique et permet à Maersk avec Algeciras, Tanger-Med et Gioia Tauro de verrouiller tous les passages stratégiques de la Méditerranée.

Cette consolidation des dynamiques portuaires de la partie orientale du bassin est également rendue visible par la montée en puissance du rôle de la Turquie. Le pays, grâce à une politique maritime active (privatisation et extension des terminaux portuaires), connait une forte augmentation de ses trafics et renforce ainsi, face à l'Egypte, un rôle de puissance maritime du bassin oriental. La région portuaire d'Istanbul s'est très vite développée au point d'offrir un complexe multi-site pour l'accueil des conteneurs grâce en particulier au terminal d'Ambarli (3 M. d' EVP en 2012) et la mise en activité prochaine du terminal de Gebse acquis par Dubaï Port World (DPW) en 2008. Le port de Mersin, en concurrence directe avec les ports égyptiens pour desservir le bassin oriental, est en cours d'extension et offrira une capacité supplémentaire de 3,9 M. d'EVP. Enfin, déjà en chantier, le port nouveau de Candarli dans la région d'Izmir devrait bientôt voir le jour et offrir une capacité de $4 \mathrm{M}$. d'EVP supplémentaires au système portuaire turc.

La région portuaire méditerranéenne connaît ainsi un dynamisme exceptionnel caractérisé par le rôle croissant des pays hors de l'Union européenne dans ces organisations nouvelles ( $c f$. figure 4). Au risque d'une surcapacité, d'autres projets sont par ailleurs en cours (en date de 2014), comme Tanger-Med II ou East Port Saïd Port qui porteront les capacités portuaires respectives à $8 \mathrm{M}$. et $13 \mathrm{M}$ d'EVP ! En Algérie, DPW prévoit de moderniser les terminaux conteneur d'Alger et de Djen Djen afin d'offrir une meilleure insertion de l'Algérie sur les marchés conteneurisés. Un complexe portuaire totalement nouveau est également prévu au cap Djenet à $50 \mathrm{~km}$ d'Alger. Enfin, la Tunisie annonce depuis plusieurs années le projet d'Enfidha, calqué sur Tanger-Med, puisqu'il s'agit de construire un port en eau profonde adossé à une vaste zone économique et logistique.

Ces fortes évolutions (trafics multipliés par six en dix ans à Port-Saïd, par cinq à Ambarli, par trois à Mersin, par deux à Ashod, Malte, Algeciras, Le Pirée...) posent la question des transformations des enjeux territoriaux dans le bassin méditerranéen. Outre les changements profonds en termes de gouvernance permis par la privatisation des terminaux et l'arrivée d'acteurs internationaux des transports et de la logistique, ce grand chambardement portuaire a en effet des conséquences régionales et locales que nous abordons dans les parties suivantes.

\footnotetext{
${ }^{4}$ Les navires dits «post-panamax » sont les plus grands capables de franchir le canal de Panama, tout comme les équipements portuaires leur étant dédiés. Le terme de «superpostpanamax » renvoie donc aux navires (et équipements dédiés) incapables, de par leurs dimensions, de franchir ledit canal.
} 
Figure 4 - Evolution des trafics conteneurs des ports méditerranéens (hors UE, 20002012, Millions d'EVP) : a) Le Sud et l'Est du bassin ; b) Les ports turcs.
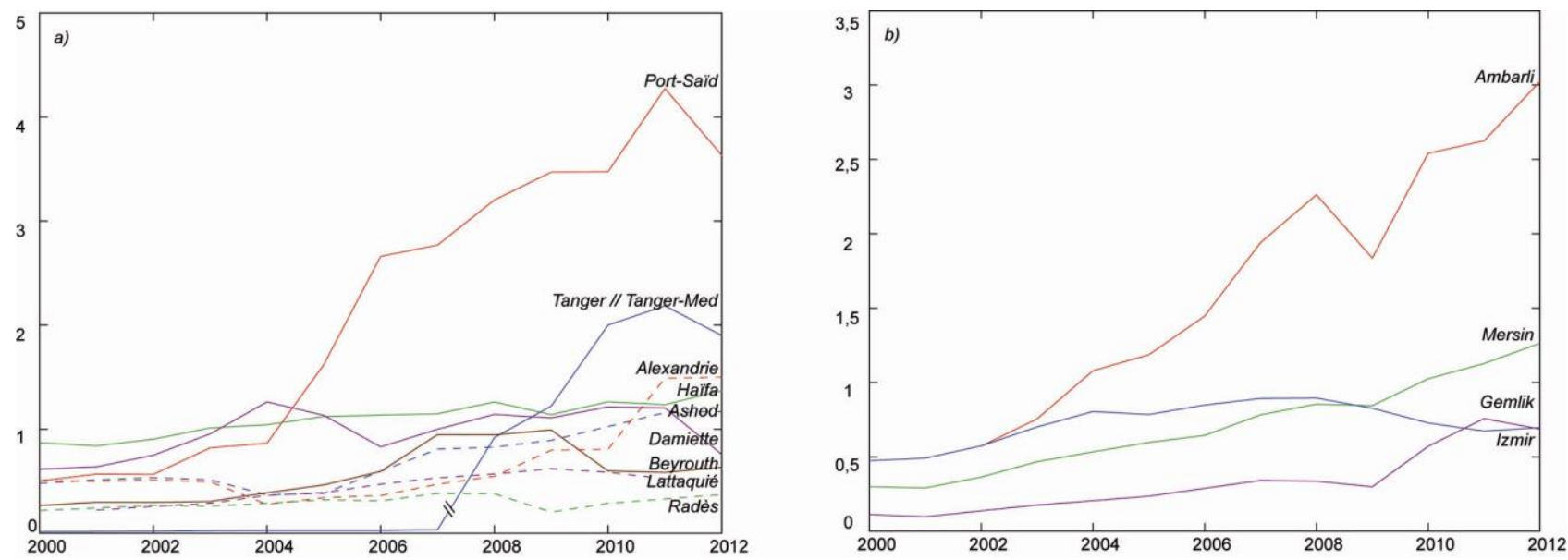

Elaborées par les auteurs à partir des données Containerisation International, ISEMAR et des autorités portuaires

\section{De la région portuaire à la région maritime}

Etonnamment, peu d'études scientifiques contemporaines proposent des analyses globales de la Méditerranée maritime et portuaire. Les travaux de Giovani Ridolfi (1999) et de Jacques Marcadon (2002) sur la conteneurisation vont dans ce sens, sans pour autant apporter une vision d'ensemble sur la manière dont le lien entre les ports et les rives s'accomplit. Dans la lignée de ces travaux, l'approche scalaire du détroit de Gibraltar et de la Méditerranée (Mareï, 2012) a permis de décrire un modèle spatial méditerranéen qui privilégie la position d'intermédiarité et qui est remarquablement illustré par l'organisation régionale du réseau méditerranéen du premier armateur mondial dans le secteur conteneurisé, Maersk ( $c f$. figure $5)$.

Figure 5 - Réseau méditerranéen de Maersk en 2011 (64 nœuds, 105 arêtes)

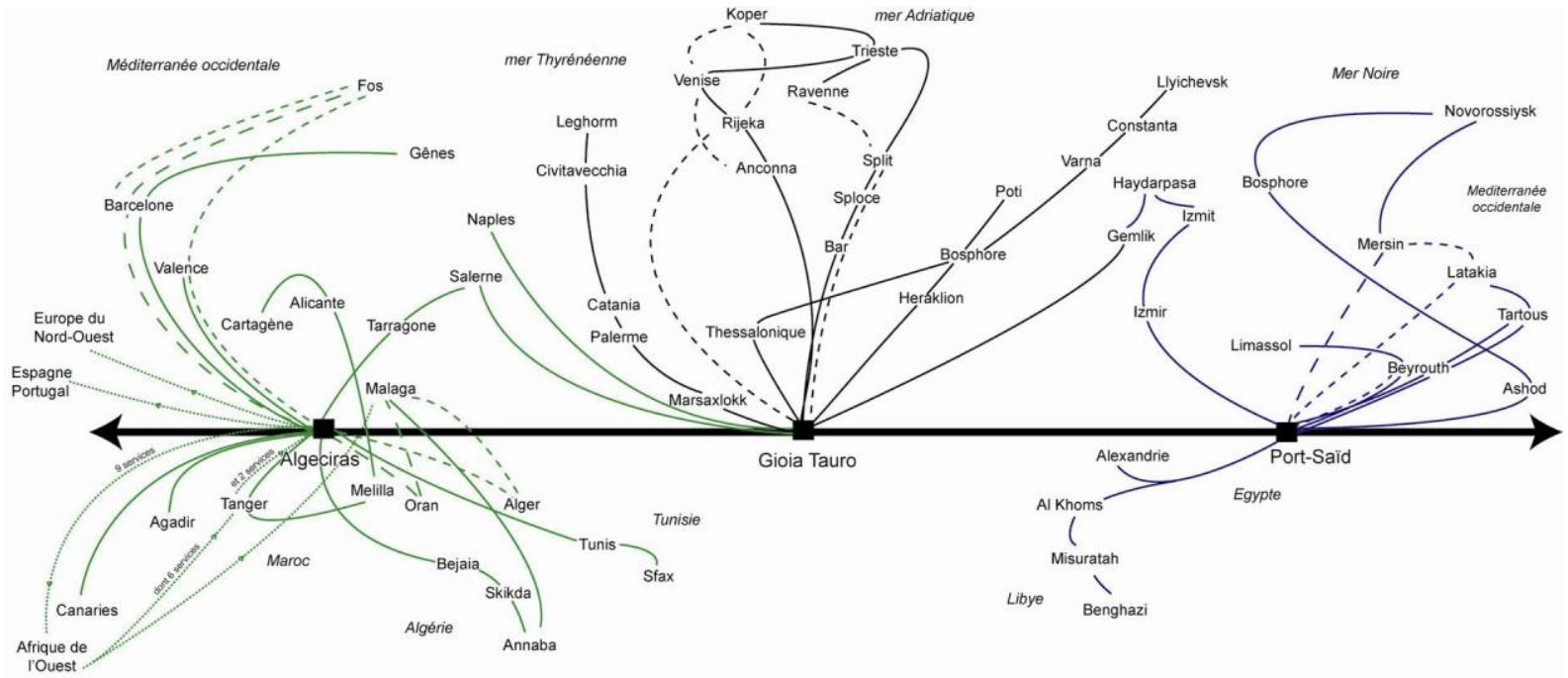

Elaboration N. Mareï, 2012. Le départ de chaque ligne s'effectue depuis un hub. Les couleurs et / ou pointillés permettent de différencier les lignes feeders. 
A partir de seulement trois hubs, 64 ports sont liés par ce réseau, montrant ainsi une Méditerranée quasiment prédisposée à une organisation en hub and spokes ${ }^{5}$ performante. Si ce schéma est symptomatique d'une organisation locale vouée au global et à la fluidité des échanges, à partir de l'analyse des réseaux méditerranéens nous nous demandons si ce fonctionnement joue un rôle prééminent dans la régionalisation de ce bassin.

Propriétés des réseaux maritimes régionaux

L'analyse des flux maritimes régionaux est effectuée en recourant aux données de mouvements quotidiens de navires publiées par Lloyd's List depuis des décennies. On construit un graphe à partir de ces flux à différentes dates-clés, à la fois pour le réseau maritime en général et pour les flux conteneurisés. Deux temporalités sont choisies : le longterme du réseau dit "global" et le court terme du réseau conteneurisé ( $c f$. tableau 1). A partir du graphe, on calcule différents indices révélant la structure d'ensemble du système de flux régional, afin d'en tirer des éléments d'interprétation de son évolution au regard des contextes successifs et des dynamiques citées plus haut de reconfiguration des échanges, et de l'adaptation ou non du bassin méditerranéen à ces dynamiques.

Tout d'abord, on constate une croissance forte dans les deux cas de la taille du graphe, mesurée par le nombre de ports et de liens entre eux. Le nombre de ports augmente sur toute la période étudiée, de même pour le nombre de lien malgré un décrochage sur la fin de la période : 85 terminaux accueillent les navires en 1890 contre 210 en 2008. La multiplication des routes dans la région explique cette croissance du graphe sur le long terme. Cette croissance est ainsi corrélée à une hausse de la densité (nombre de liens par port en moyenne). Au niveau du nombre d'escales, en revanche, on constate une baisse du nombre de celles-ci en Méditerranée d'environ 80\% entre 1925 et 1960, baisse qui se démarque fortement de la très légère baisse au niveau mondial $(-1 \%)$. Ceci est directement lié aux effets à court et moyen terme de la crise de Suez ayant provoqué la fermeture du canal sur les périodes 19561957 et 1967-1975. Pour le reste, la tendance méditerranéenne est en phase avec le niveau mondial, soit un tassement du nombre de liens et d'escales entre 1980 et 2008.

La complétude (part des liens observés dans le maximum de liens possibles) diminue pour les deux réseaux analysés. La croissance moindre du nombre de ports par rapport au nombre de liens entre eux est un effet direct des économies d'échelles réalisées par les armateurs (course au gigantisme des navires, rationalisation et concentration des escales) pour optimiser leurs services, tant dans le secteur des conteneurs que celui des vracs ${ }^{6}$ liquides (pétrole, gaz). énergétiques ou de biens manufacturés, et expliquent donc cette diminution de la connectivité. C'est d'autant plus le cas en Méditerranée puisque la plupart des ports de transbordement pour les conteneurs y sont également de grands ports vraquiers pour les hydrocarbures (ex : Tarente, Algésiras, Cagliari), ce qui accentue d'autant plus cet effet de concentration en un nombre restreint de places portuaires multifonctionnelles.

Le réseau conteneurisé illustre parfaitement bien cette tendance, car sa complétude baisse régulièrement sur la période étudiée. En effet, comme souligné précédemment, le déploiement

\footnotetext{
${ }^{5}$ Un réseau en $h u b$ and spokes est un réseau en étoile à partir d'une plate-forme principale. Des navires de plus petites tailles (feeders) chargent les marchandises au hub pour distribution vers des ports secondaires. Le terme spokes qualifie donc les arrêtes secondaires.

${ }^{6}$ Le vrac est une catégorie de marchandises transportées en gros, le plus souvent non conteneurisées, concernant des matières premières (charbon, minerais), pondéreux, ou autres denrées volumineuses (céréales). On distingue généralement le vrac sec (solid ou dry bulk) du vrac liquide (liquid bulk).
} 
des stratégies de hub-and-spokes en Méditerranée coïncide avec le milieu des années 1990. On peut donc supposer que le réseau devient de plus en plus optimal (avec une meilleure capacité logistique) et de moins en moins "complet" - le réseau complet se définissant par le maximum de liens possibles entre tous les ports, le but des armateurs étant de rationaliser leurs escales en les concentrant autour de grandes plates-formes de redistribution. Enfin, le coefficient moyen de clustering (probabilité moyenne que les ports voisins d'un port donné soient également connectés entre eux) connaît une phase d'augmentation puis de diminution avant et après 1960 (réseau global), et une augmentation constante entre 1996 et 2011 (réseau conteneurisé). Cela traduit la mise en place d'un maillage régional avant la concentration évoquée précédemment, même si l'on pouvait s'attendre à ce que le réseau conteneurisé soit plus «optimal » que le réseau global, notamment sur la période récente. Il semble en effet que, en Méditerranée, rationalisation et densification du réseau (maillage) aillent de pair, du fait de la coïncidence de logiques à la fois globales - concentration des flux via des hubs - et locales - renforcement des liens de proximité, notamment via le transport maritime de courte distance.

La cartographie des flux dits "majeurs" permet d'affiner ces propos par l'identification des principaux pivots et sous-systèmes articulant la région maritime méditerranéenne ( $c f$. figure 6). Au niveau méthodologique, et uniquement dans le cas du réseau conteneurisé, la simplification du réseau par les flux majeurs permet de ne retenir, pour chaque port, que son flux le plus volumineux vers un autre port. La conséquence en est la partition du réseau en plusieurs composants connexes et la valorisation des ports ayant un rôle central dans le système régional.

Tableau 1 - Propriétés des réseaux méditerranéen et mondial

\begin{tabular}{|c|c|c|c|c|c|c|c|c|c|}
\hline \multirow[t]{2}{*}{ Indicateur } & \multirow[t]{2}{*}{ Région } & \multicolumn{5}{|c|}{ Réseau global* } & \multicolumn{3}{|c|}{$\begin{array}{c}\text { Réseau } \\
\text { conteneurisé** }\end{array}$} \\
\hline & & 1890 & 1925 & 1960 & 1980 & 2008 & 1996 & 2006 & 2011 \\
\hline \multirow{2}{*}{$\begin{array}{c}\text { Nb. } \\
\text { d'escales }\end{array}$} & Med. & 2112 & 2872 & 565 & 1011 & 887 & 1136 & 2849 & 4165 \\
\hline & Monde & 10765 & 22661 & 21055 & 19893 & 15289 & 12497 & 28969 & 40127 \\
\hline \multirow{2}{*}{ Nb. Liens } & Med. & 168 & 296 & 351 & 673 & 611 & 221 & 373 & 640 \\
\hline & Monde & 5055 & 9746 & 10627 & 11549 & 8448 & 2141 & 3888 & 4950 \\
\hline \multirow{2}{*}{ Nb. Ports } & Med. & 85 & 126 & 123 & 175 & 210 & 51 & 73 & 100 \\
\hline & Monde & 1232 & 1460 & 1453 & 1571 & 1918 & 542 & 740 & 853 \\
\hline \multirow{2}{*}{ Densité } & Med. & 1.976 & 2.349 & 2.853 & 3.845 & 2.909 & 4.333 & 5.110 & 6.400 \\
\hline & Monde & 4.103 & 6.675 & 7.314 & 7.351 & 4.405 & 3.950 & 5.254 & 5.803 \\
\hline \multirow{2}{*}{ Complétude } & Med. & 0.047 & 0.038 & 0.047 & 0.044 & 0.028 & 0.173 & 0.142 & 0.129 \\
\hline & Monde & 0.007 & 0.009 & 0.010 & 0.009 & 0.005 & 0.015 & 0.014 & 0.014 \\
\hline \multirow{2}{*}{ Clustering } & Med. & 0.113 & 0.203 & 0.299 & 0.252 & 0.159 & 0.401 & 0.506 & 0.532 \\
\hline & Monde & 0.335 & 0.409 & 0.389 & 0.298 & 0.174 & 0.450 & 0.501 & 0.483 \\
\hline
\end{tabular}

Ainsi, la région maritime méditerranéenne apparaît plutôt polycentrique, c'est-à-dire animée par plusieurs grands ports se partageant l'essentiel des connections importantes. Cela n'exclut pas des changements majeurs, comme l'émergence de Gioia Tauro en Calabre aux dépens du Pirée ou encore de Limassol à Chypre. On observe également des fluctuations dans 
l'appartenance des grands ports aux sous-ensembles du réseau. Marsaxlokk et Algeciras, à l'écart du système régional en 1996 de par leur rôle avant tout global dans les flux maritimes, sont réintégrés en 2006 dans le composant principal (rouge). Pourtant, Algeciras intègre en 2011un autre sous-ensemble composé surtout de ports espagnols. D'autres sous-ensembles locaux émergent, comme en Adriatique autour des ports de Venise/Koper, et dans le Bosphore, rassemblant de nombreux ports turcs autour d'Ambarli (Istanbul). Ces dynamiques indiquent la concomitance des logiques de proximité et des logiques exogènes dans l'évolution des flux maritimes régionaux, comme suggéré dans le Tableau 1.

\section{Dynamiques réticulaires et liens régionaux}

La Méditerranée maritime et portuaire demeure un espace très hétérogène sur lequel ne s'est pas structurée une véritable façade maritime qui serait le miroir d'une économie pourtant fortement tournée vers la mer. Cependant, le bassin subit actuellement des bouleversements importants, d'ordres globaux et régionaux, qui redessinent et complexifient la carte de cette interface à la fois eurafricaine et mondiale. Des nœuds nouveaux émergent et ont tendance à centraliser des dynamiques sous-régionales (Debrie et al., 2005). Pourront-ils à l'avenir consolider des polarités nouvelles susceptibles d'alimenter le système en constitution ? La question reste posée comme un enjeu d'avenir. Dès lors, deux grandes dynamiques complémentaires contribuent à la construction de la région maritime :

1- Le renforcement des réseaux conteneurisés autour des ports de pertuis c'est-à-dire ceux situés sur les passages maritimes (Mareï, 2013), selon une double dynamique :

- Une logique purement réticulaire qui place les ports bénéficiant d'une situation optimale sur la grande route du commerce eurasiatique dans le réseau maritime d'un opérateur de taille mondiale. Leur fonction principale devient celle d'une plate-forme de redistribution des conteneurs, à l'échelle régionale (Port-Saïd, Gioia Tauro, Malte) ou mondiale (Algeciras).

- Une logique régionale permise par le renforcement des liens de proximité permettant d'une part l'intégration des hubs dans le réseau régional, et d'autre part la naissance de dynamiques sous-régionales qu'on retrouve autour des trois portes du bassin (Gibraltar, Suez et Bosphore), ou des ports de l'Adriatique. Ces dynamiques plus intégrées sont en partie imputables à des politiques nationales et régionales de développement favorisant les échanges intra-bassins (renforcement des filières fruits et légumes au Maroc, le textile en Turquie, les hydrocarbures en Algérie et en Egypte, les autoroutes de la mer du programme européen Marco Polo, ...).

2- La consolidation du maillage global du bassin ( $c f$. augmentation globale du coefficient de clustering) peut s'expliquer par la diversité des relations maritimes intra-bassins (flux rouliers relais au transport international routier entre les deux rives, passagers, énergétiques). L'exemple des lignes rouliers-passagers (ro-pax) est intéressant car il s'agit d'un système anciennement implanté en Méditerranée occidentale et en développement à l'Est par les autoroutes de la mer au départ des ports turcs et à destination de l'UE par exemple. Chaque année, près de 8 millions de passagers empruntent les lignes ro-pax ouest-méditerranéennes dont 5 millions passent par le détroit de Gibraltar. Il s'agit de maghrébins résidents en Europe (MRE) qui voyagent saisonnièrement, de touristes, et même des travailleurs saisonniers dans le domaine agricole en particulier. Ces lignes servent également à l'import-export entre les deux 
rives de la Méditerranée puisque les navires de la traversée embarquent, en plus des passagers, des camions de marchandises, parfois même des conteneurs. Un groupe comme Grimaldi Lines, leader italien des trafics rouliers, utilise par exemple des navires pouvant transporter en plus des passagers et des véhicules, 400 à 800 conteneurs sur des lignes régulières reliant les grands ports de l'ouest du bassin ( $c f$. figure 7). Ce système roulier, fortement implanté sur le bassin échappe aux stratégies de rentabilité des dessertes de la route Europe-Asie et peut alors offrir un réseau avec une plus grande connectivité Nord-Sud grâce à un maillage moins hiérarchisé que celui des réseaux conteneurisés (Mareï, 2012).

Figure 6 - Principaux flux conteneurisés méditerranéens (1996-2011)

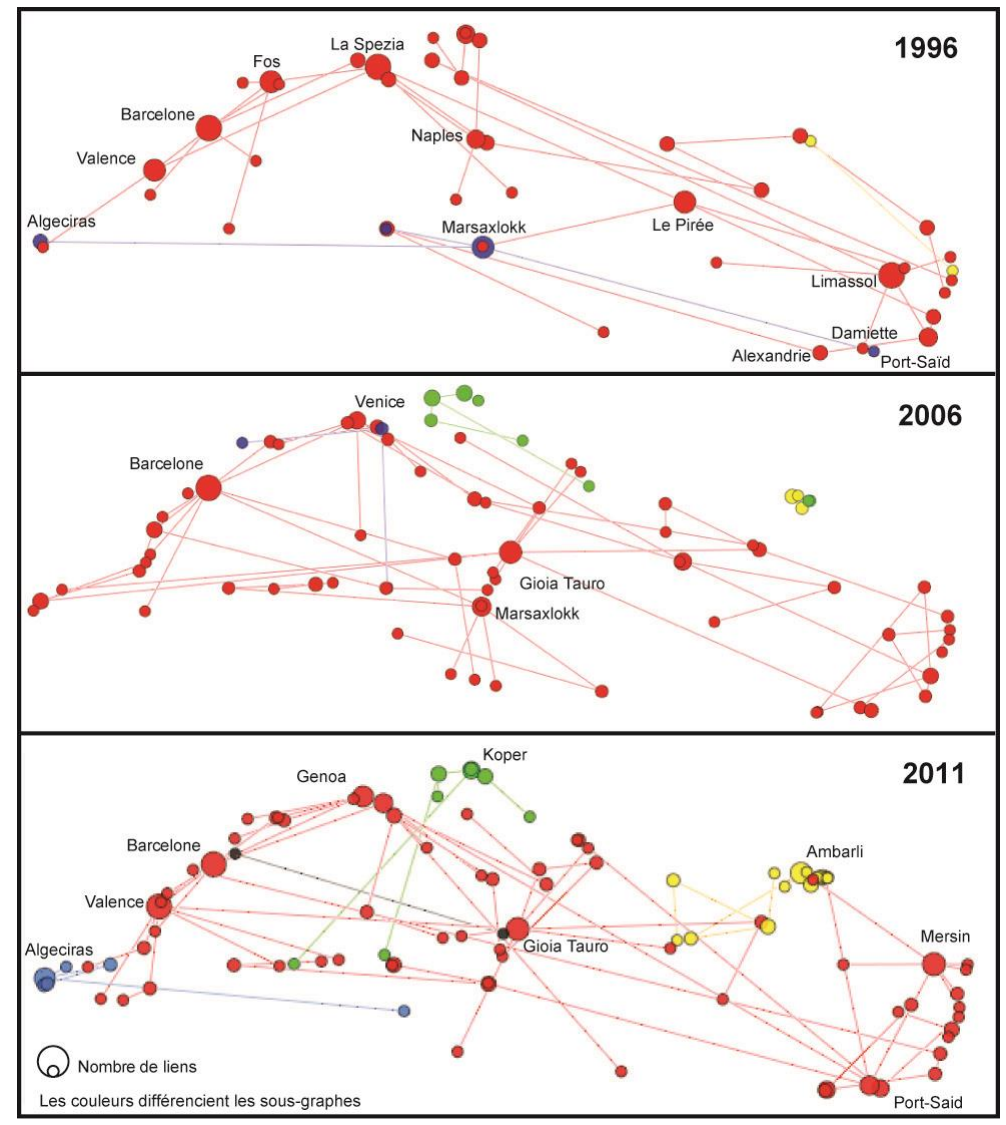

Elaborée par les auteurs d'après Lloyd's List 
Figure 7 - Réseaux rouliers de la Méditerranée Occidentale (2011).

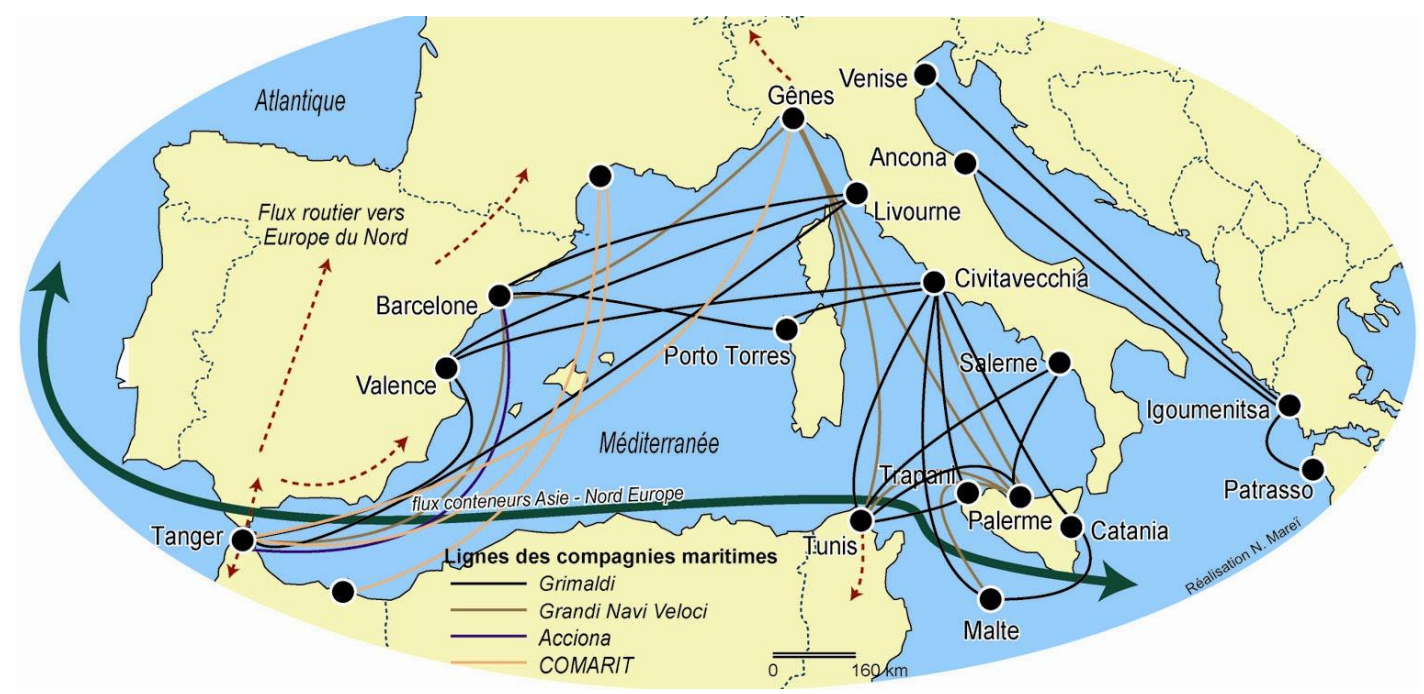

Elaboration N. Marë̈, 2012 d'après les compagnies maritimes

\section{L’enracinement territorial des échanges mondialisés}

L'analyse de la manière dont circulent et sont captées les marchandises en Méditerranée permet de décrire un système global voué à la fluidité du réseau et entretenu par des intérêts extérieurs. Les réflexions sur l'enracinement territorial se posent alors en termes de fixité (Hesse et Rodrigue, 2004) et de fécondité (Steck, 2009). La fixité qui se rattache à ce qui freine la circulation n'est pas antinomique de la fécondité qui peut être définie comme la capacité du territoire logistique à produire des externalités positives (création de nouveaux flux, émergence de centralités nouvelles). Les deux notions sont liées car c'est dans l'évolution des contextes locaux (réglementation, foncier, rapports de force des parties prenantes...) que peut naître davantage de porosité entre réseaux et territoires. Ces considérations nous amènent ainsi à nous interroger sur la traduction locale des échanges mondialisés.

Le nouveau port marocain Tanger-Med est symptomatique de cette tension mondial / local qui existe dans - le bassin (Mareï, 2013). Il est l'archétype de la modernisation portuaire au Sud $\mathrm{du}$ bassin et du positionnement stratégique d'un nouveau hub pour capter les trafics de transbordement, en concurrence avec Algeciras principalement. Le nouveau port a attiré les grands manutentionnaires et opérateurs internationaux (APM Terminal, Maersk, CMA-CGM, PSA) et prévoit d'étendre ses capacités à $8 \mathrm{M}$. d'EVP au terme des travaux de Tanger-Med II. Mais le projet Tanger-Med est plus ambitieux. Il consiste en réalité, grâce à une panoplie de zones d'activité, en une opération d'aménagement du territoire de la région Tanger-Tétouan. L'objectif est d'attirer des investissements étrangers et d'impulser un développement régional nouveau par la modernisation de l'ensemble du système de transport et par l'arrimage à la fois aux flux maritimes internationaux et régionaux. Si le succès commercial de l'opération est incontestable, le bilan n'est pas aussi spectaculaire sur le plan du développement local. Il est vrai que la zone franche Tanger Free Zone s'est lotie rapidement suite à la décision de création de Tanger Med : cette zone comprend 300 ha de zone industrielle et 65 ha de zone logistique, et elle accueille aujourd'hui près de 400 sociétés générant 40000 emplois. Il est 
vrai aussi que la décision de Renault d'implanter une usine à Melloussa (dès 2008) est directement liée à Tanger Med et que cette décision peut avoir d'indéniables effets d'entraînement (on parle de 6000 emplois). La zone franche logistique Medhub, à peine commercialisée, connaît déjà ses premiers succès en 2008, dont la décision de CILSA, société de la zone logistique du port de Barcelone, de s'implanter sur 5 ha. La législation leur est extrêmement favorable : pas de droits de douane, pas de TVA, pas d'impôts sur les sociétés pendant cinq ans, pas de restriction en matière de rapatriement de capitaux ou de transactions en devises étrangères, le tout protégé par un préavis de vingt ans en matière de modification de la réglementation (BIT, 2003 ; Bost, 2007). Mais il faut aussi rappeler que les effets des zones franches sont limités par les principes mêmes de leur développement : faible intégration socio-économique avec les territoires d'accueil, extraversion des flux de marchandises. Il y a donc un pas à franchir entre l'implantation d'une infrastructure nouvelle et l'enracinement territorial.

Malgré le succès en demi-teinte de Tanger-Med, la multiplication des travaux de modernisation des infrastructures portuaires et les projets de ports nouveaux en Méditerranée montrent le désir de dupliquer ce modèle. Ces opérations sont accompagnées d'une ouverture des pratiques économiques (réformes portuaires, appel à l'investissement étranger) qui participent à l'attractivité internationale du bassin mais qui peut également servir le développement interne, comme peut le suggérer la progression globale (ro-ro, conteneurs, pétrole) des trafics intra-bassin et les projections de croissance de l'ordre de $18 \%$ (AGAM 2013, LMIU, 2008).

La normalisation des pratiques portuaires (modernisation et privatisation, augmentation des capacités de stockage, spécialisation) est porteuse de porosités nouvelles entres les rives de la Méditerranée : à l'interconnexion Est-Ouest, objectif premier des opérateurs globaux installés en Méditerranée, répondent des dynamiques nouvelles de proximité (liens intra-bassins nouveaux, autoroutes de la mer, création ou renforcement de filières de production). La modernisation du système portuaire en dehors de l'Union européenne (Maroc, Egypte, Turquie) apparaît donc comme un facteur d'intégration régionale et économique basée sur les échanges commerciaux. Il s'agit là du modèle de développement de l'Asie orientale, dont on sent les prémices timides en Méditerranée et surtout la fragilité. Au delà de l'instabilité créée par la crise économique mondiale, c'est la faible intégration Nord-Sud qui peut être pointée du doigt. En effet, la relation de l'Europe à son Sud est anormalement faible comparée aux autres grandes régions Nord-Sud : l'Europe ne réalise que 2,3\% de ses investissements au Sud de la Méditerranée alors que les Etats-Unis et le Japon dirigent plus de $20 \%$ de leurs investissements directs étrangers (IDE) vers « leur Suds » respectifs (Telle, 2009). Les efforts à réaliser dans le sens d'une régionalisation des investissements sont alors incontestables.

Il ne s'agit pas ici de discuter les difficultés du projet d'union pour la Méditerranée, affaibli certes depuis les révolutions arabes mais en panne bien avant cette situation. Simplement, il est certain que ce projet demeura un vœu pieux s'il n'est pas basé sur un modèle de développement plus intégré, reposant sur des bases concrètes comme la création de filières régionales. Les infrastructures et les réseaux pouvant supporter ces échanges existent sur le bassin. De ce point de vue, la Méditerranée est donc un espace fonctionnel, qu'il est urgent d'alimenter. Du succès d'une telle stratégie dépend l'équilibre général du bassin, se traduisant notamment par des relations détendues et fécondes entre ses deux rivages. 


\section{Conclusion}

Les enjeux spatiaux du bassin maritime méditerranéen sont de la plus haute importance, à toutes les échelles de leur observation : au niveau mondial d'abord, où les opérateurs se positionnent pour avoir les meilleures places; au niveau national ensuite, où la création de hubs permet de concevoir des stratégies de développement, en respectant le délicat paradoxe de faire de ces territoires portuaires des sites propices à l'investissement international ; au niveau régional enfin, où la brutalité des impacts doit être maîtrisée pour éviter d'en subir les externalités négatives. En effet, si la régionalisation se consolide dans le bassin, la relation réseau-territoire est plus complexe. Le réseau crée d'emblée un rapport distant au territoire qui ne pourra être contourné que par davantage d'intégration Nord-Sud d'une part, et d'intégratoion littoral-arrière-pays d'autre part, la pénétration à l'intérieur des terres des circulations mondiales étant un autre enjeu pour les riverains du bassin. Les perspectives de développement de la région maritime et portuaire sont alors dans le renforcement des fonctions logistiques locales afin d'offrir un meilleur ancrage à l'arrière-pays, et espérer de réels effets induits en matière d'ouverture économique de la région.

La problématique de la porosité entre les réseaux et les territoires est fortement liée à celle de l'intégration régionale. En définitive, nous venons d'analyser un mécanisme global de régionalisation économique par les réseaux maritimes. Ce dernier est intégrateur à l'échelle régionale car les pays du Sud participent au processus, sans pour autant être gage de développement pour ces pays car les retombées économiques ne sont pas forcément locales. Le passage au stade de l'intégration régionale, et donc à la fabrique d'une régionalité nouvelle, pour reprendre l'idée de regioness, est plus délicat. Il résulte d'une conjugaison de dynamiques locales, globales, de réseaux, de territoires, d'évolutions politiques, économiques, sociales inscrite dans la durée et qui ne se superpose pas forcément avec les territoires administrés par les Etats. L'intégration régionale s'alimente aussi d'une régionalisation par «le bas» (Tarrius, 2007) issues de réseaux plus «discrets» (Pliez, 2007), ceux des entrepreneurs locaux, des migrants, des réseaux de la communication qui œuvrent le long des espaces relais de la mondialisation ou à côté (Lombard et Ninot, 2012) et qui participent avec les réseaux macro-économiques à la production d'une nouvelle géographie méditerranéenne.

\section{Bibliographie}

Agence d'urbanisme de l'agglomération marseillaise (AGAM), 2013. Atlas des villes portuaires du sud et de l'Est de la Méditerranée, Synthèse, [en ligne]

Amin, A., 2004. Regions unbound: towards a new politics of place. Geografiska Annaler: series B, 86 (1): 33-44.

Beckouche, P., 2008. Les régions Nord-Sud, Paris, Belin.

Bentley J., 1999. Sea and Ocean Basins as Frameworks of Historical Analysis. Geographical Review, Vol. 89, no. 2, Special Issue Oceans Connect, 89 (2): 215-224.

Bureau International du Travail (BIT), 2003. Emploi et politique sociale dans les zones franches d'exportation. 286e session de la commission de l'emploi et de la politique sociale, Genève, [en ligne] 
Bost F., 2007. Les zones franches interfaces de la mondialisation. Annales de Géographie, $\mathrm{n}^{\circ} 658, \mathrm{p}$ 563-585.

Carroué, L., 2007. Géographie de la mondialisation, Paris, Armand Colin.

CNUCED, 2008. Etude sur les transports maritimes. New York : Publication des Nations Unies, [en ligne]

Claval P., 1968. Régions, nations, grands espaces. Géographie générale des ensembles territoriaux, Paris, Marie-Thérèse Génin.

Debrie J., Eliot E. et Soppé M., 2005. Un modèle transcalaire des nodalités et polarités portuaires : exemple d'application au port de Hambourg. Dans Mappemonde, 79, 12 pages, [en ligne].

Ducruet, C., Mohamed-Chérif, F.Z., Cherfaoui, N., 2011. Maghreb port cities in transition: the case of Tangier, Portus Plus, vol. 1, $\mathrm{n}^{\mathrm{o}}$ 1, (en ligne)

Ducruet C., 2009. Port regions and globalization. In: Ports in Proximity: Competition and Coordination among Adjacent Seaports, Notteboom et al. (Eds), Aldershot, Ashgate, pp.4153.

Ducruet, C. 2008. Typologie mondiale des relations ville-port. Cybergeo : European Journal of Geography, [en ligne]

Fleming D.K. et Hayuth Y., 1994. Spatial characteristics of transportation hubs: centrality and intermediacy. Journal of Transport Geography, 2(1), pages 3-18.

Frémont A., 2007. Global maritime networks. The case of Maersk. Journal of Transport Geography, 15: 431-442.

Gipouloux F., 2009. La Méditerranée asiatique. Villes portuaires et réseaux marchands en Chine, au Japon et en Asie du Sud-Est, XVIe - XXIe siècle. CNRS éditions, Paris.

Girault C., 2009. Intégrations en Amérique du Sud, Paris, Presses de la Sorbonne nouvelle.

Grataloup Ch.,2007. Géohistoire de la mondialisation. Le temps long du Monde. Paris: Armand Colin.

Hesse M. et Rodrigue J., 2004. The transport geography of logistics and freight distribution, Journal of Transport Geography, 12(3), pages 171-184.

Hettne B. et Söderbaum F., 1998. The New Regionalism Approach. Politeia, 17(3), 6-22.

Hettne B. et Söderbaum F., 2000. Theorizing the Rize of Regionness. New Political Economy, $5(3), 457-474$.

Jones M. et Paasi A., 2013. Guest Editorial: Regional World(s): Advancing the Geography of Regions, Regional Studies, 47: 1-5. 
Lemarchand A., Joly O., 2009. Regional integration and maritime range. In: T. E. Notteboom, C. Ducruet, \& P. W. de Langen (Eds.), Ports in proximity: Competition and coordination among adjacent seaports (pp. 87-99). Aldershot: Ashgate.

Lewis M.W., Wigen K., 1999. A maritime response to the crisis in area studies, The Geographical Review, 89(2): 161-168, Special Issue Oceans Connect.

Lloyd's Marine Intelligence Unit (LMIU), 2008. Étude des flux du transport maritime en mer Méditerranée, REMPEC, [en ligne]

Lombard J. et Ninot O., 2012. Des mobilités aux transports. Regards croisés en Afrique de l'Ouest, EchoGéo, 20.

Marcadon J., 2002. Géographie portuaire de l'espace euro-méditerranéen. Méditerranée, 98(1), pages 55-66.

Mareï N., 2014. La Méditerranée sous le regard de la conteneurisation, Note de Synthèse de l'ISEMAR 163, [en ligne]

Mareï N., 2013. Le détroit de Gibraltar, Des réseaux mondiaux aux enjeux locaux de développement, Géotransports, 1-2, pp 205-220, [en ligne]

Mareï N., 2012. Le détroit de Gibraltar, Porte du Monde, Frontière de l'Europe. Analyse et perspectives de territorialité d'un espace de transit. - Thèse de doctorat en géographie, Université de Nantes.

Mohamed-Chérif, F.Z, Ducruet, C., 2011. Les ports et la façade maritime du Maghreb : entre intégration régionale et mondiale, Mappemonde, 101, [en ligne]

Ohmae K., 1996. De l'Etat-nation aux Etats-régions. Dunod.

Pliez O., 2007. Des jeans chinois dans les rues du Caire, ou les espaces discrets de la mondialisation, Mappemonde, 88 (4).

Richard Y., 2010. L'Union européenne et ses voisins orientaux. Contribution à l'étude des intégrations régionales dans le monde. 440 pages. Habilitation à Diriger des Recherches, Université Paris 1 Panthéon-Sorbonne.

Ridolfi G., 1999. Containerisation in the Mediterranean: between global ocean routeways and feeder services. GeoJournal, 48(1), pages 29-34.

Rodrigue J.P., Comtois C. et Slack B., 1997. Transportation and spatial cycles: evidence from maritime systems. Journal of Transport Geography, 5(2), pages 87-98.

Steck B., 2009. Transport et développement, in M. Brocard (dir.), Transports et territoires, enjeux et débats, Paris, Ellipses, p. 125-156.

Tarrius A., 2007. La remontée des Sud: Afghans et Marocains en Europe méridonale. Editions de l'Aube. 
Telle S., 2009. L’Union pour la Méditerranée : un héritage contrasté mais une continuité indispensable. Outre-Terre, 23(3), pp 19-29.

Vidal de la Bache, P., 1903. Tableau géographique de la France, Paris: Hachette

Vigarié A., 1990. Economie maritime et géostratégique des océans. Caen : Paradigme.

Vigarié A., 1964. Les grands ports de commerce de la Seine au Rhin: leur évolution devant l'industrialisation des arrièe-pays, Paris: S.A.B.R.I. 